\title{
Recent Progress on Bio-mechanical Energy Harvesting System from the Human Body: Comprehensive Review
}

\author{
Mohankumar $\mathrm{V}^{1}$ \\ Research Scholar, Department of Electronics and \\ Communication Engineering \\ VTU, Belagavi, Karnataka, India
}

\author{
G.V. Jayaramaiah ${ }^{2}$ \\ Professor and Head, Department of Electrical and \\ Electronics Engineering, Dr. Ambedkar Institute of \\ Technology, Bengaluru-560056, Karnataka, India
}

\begin{abstract}
Energy harvesting is a powerful technique to produce clean and renewable energy with better infrastructure improvement. The exhaustive review of recent progress and development in bio-mechanical energy harvesting (BMEH) techniques from human body is discussed in this manuscript. The BMEH from the human body is categorized into three parts, namely, piezoelectric energy harvesting (PEEH), triboelectric energy harvesting (TEEH), and Electro-magnetic Energy harvesting (EMEH). Each energy harvesting system is discussed with working principles with mathematical equations; each energy harvesting progress is discussed with a few work demonstrations. The applications of each energy harvesting from the recent research work are addressed in detail. The summary of each energy harvester from the human body or motion with advantages, limitations, performance metrics, current methods, and implemented human body parts are highlighted with Tabulation. The critical challenges/issues with possible solutions are also discussed.
\end{abstract}

Keywords-Bio-mechanical; energy harvesting; electromagnetic; human-body; piezoelectric; triboelectric

\section{INTRODUCTION}

Renewable energy resources are necessary to solve most of the significant challenges for sustainable development. The power is generated using the most commonly used energy resources like natural gas, petroleum, Hydraulic, nuclear energy, and coal. Energy harvesting is one of the essential processes to produce clean, renewable energy and improves infrastructure development. The energy harvesting (EH) process receives unused energy and is transformed into the most usable energy resource. The commonly used and available energy resources for the energy harvesting process are kinetic, solar, thermal, wind, hydro energies. The EH system is used to recharge batteries, increase the life span of electronic devices, and battery replacement in a low-power system. Firstly, the batteries are recharged easily by using energy harvesting. Next, the electronics devices' life cycle can be prolonged using an energy harvesting system. Lastly, the low-power circuits generally use $1 \mathrm{~mW}$ total power, so integrate the energy harvesting method in small integrating circuits to consume less the $10 \mu \mathrm{W}-100 \mu \mathrm{W}$ total power [1-2].

The Human body needs energy and performs its day-to-day activities by consuming more and more energy. Nowadays most of the peoples use wearable devices and essence of home appliances while doing their activities. Metabolism produces enormous energy in the human body and is a prime resource for harvesting energy. Typical movements like walking, running, finger movement, and many other movements are performed daily to generate power in the human body. The heat, bio-chemical potentials, and limb movements produce the central part of the energy in the human body. Like lower limb movement, the body movement produces more energy because it has higher torque than other body parts [3-4]. The energy harvested from the human body is not affected much by dayto-day human life activities. Because of this, most of the current research is focused on human-body energy harvesting. The human body dissipates more energy every day. Even though the utilization ratio is minimal for the proportion of human energy, only micro to milliwatts of power are converted. Therefore, the Harvested power from the human body is insufficient to meet the electricity demand of people's daily Usage [5-6]. The human body energy resources are used in many ultra-lower applications with high efficiency. The various wearable, portable electronic devices, implantable medical devices are instantiated into people's daily Usage by using harvested human power. People use the human's energy resources for daily charging, battery replacement, replacement of implantable electronic devices, and other activities in an essential manner. The energy resources from the human body provide a reliable, flexible, safe environment and no side effects from environmental and space conditions. The human body energy is divided into three forms: Thermal, chemical, and mechanical energies. There is a need for an effective energy harvesting mechanism based on humans' biomechanical movement. The developments of a new form of electronic devices rely on energy harvesting technologies. Recent researches have improved the biomechanical energy harvesting system with suitable changes in the design and configurations of piezoelectric $\mathrm{EH}$, triboelectric $\mathrm{EH}$, and electromagnetic $\mathrm{EH}$ systems. The design and configuration are done by considering the particular energy harvesting technology challenges and achieving high energy harvesting. However still, various challenges exist in biomechanical energy harvesting techniques [7-9].

This review has made an exhaustive summary and analysis of the recent development of biomechanical energy harvesting from the human body. This work aims to provide insight into recent work on human-centric biomechanical energy 
harvesting systems using Piezoelectric, Triboelectric, and Electromagnetic modules. Section II discusses the energy distribution parameters in the human body. The working principles of the biomechanical energy harvesting systems are addressed in Section III. The recent progress of the biomechanical energy harvesting systems is analyzed in detail with a comparative study in Section IV. The challenges and possible solutions are highlighted in Section V. Lastly, the complete work is concluded with futuristic suggestions are incorporated in Section VI.

\section{ENERGY DisTRIBUTION IN HUMAN BODY}

The human body consumes and releases different forms of energy. For example, every movement and even breath produce energy all the time in the human body. The thermal, chemical and mechanical energies are the available potential energy resources in the human body. The potential energy resources and energy dissipation in the human body for different activities are represented in Fig. 1 [5, 7].

Every day human body consumes more amount of energy from metabolism to maintain the constant body -temperature. The thermal energy produced in the form of heat is exchanged between the body and its surroundings. The other forms of thermal energy are released with the help of respiration or breathing, evaporation heat produced from the sweat in the skin. Chemical energy is produced in the form of water (H2O), glucose, and lactic acid. The Human body digest and absorbs the food and is converted in the form of glucose. The glucose in blood glucose is distributed into other parts of the body to regenerate the energy in one other form. Additionally, the muscles produce the lactic acid by performing the exercise operations, and a few amounts of lactate decomposed in the muscle itself. The sweat is also produced while performing the exercise operations in the form of the substance of water. The mechanical energy is produced in the form of body movement, Heartbeat, Respiratory movement. The Limbs movements like steeping, arms turning, foot lifting, tapping, and other movements consume and release the mechanical energy. In addition to that, heartbeat, blood vessels relaxation and contraction, and respiratory movements are also consuming and releasing mechanical energy. The muscle's relaxation and contraction produce more mechanical energy to perform additional activities with continuous energy transmission.

The energy resources are evaluated further by analyzing the power utilization of different energy flows in the human body. The human body's energy is converted into electricity, which is less than the total amount of energy utilized in the typical energy flow of each human body's part. This is the primary concept and is discussed further with different energy harvesting methods for the human body. The human energy resources and their recent energy harvesting methods are represented in Fig. 1. The thermal energy is harvested using a pyroelectric and thermo-electric generator. Similarly, the chemical energy is harvested using a Hydro-voltaic effect generator and biofuel cell. Lastly, the mechanical energy is harvested using Piezoelectric, triboelectric and electromagnetic generators. In this article, the bio-mechanical energy harvesting techniques are discussed further with different applications.

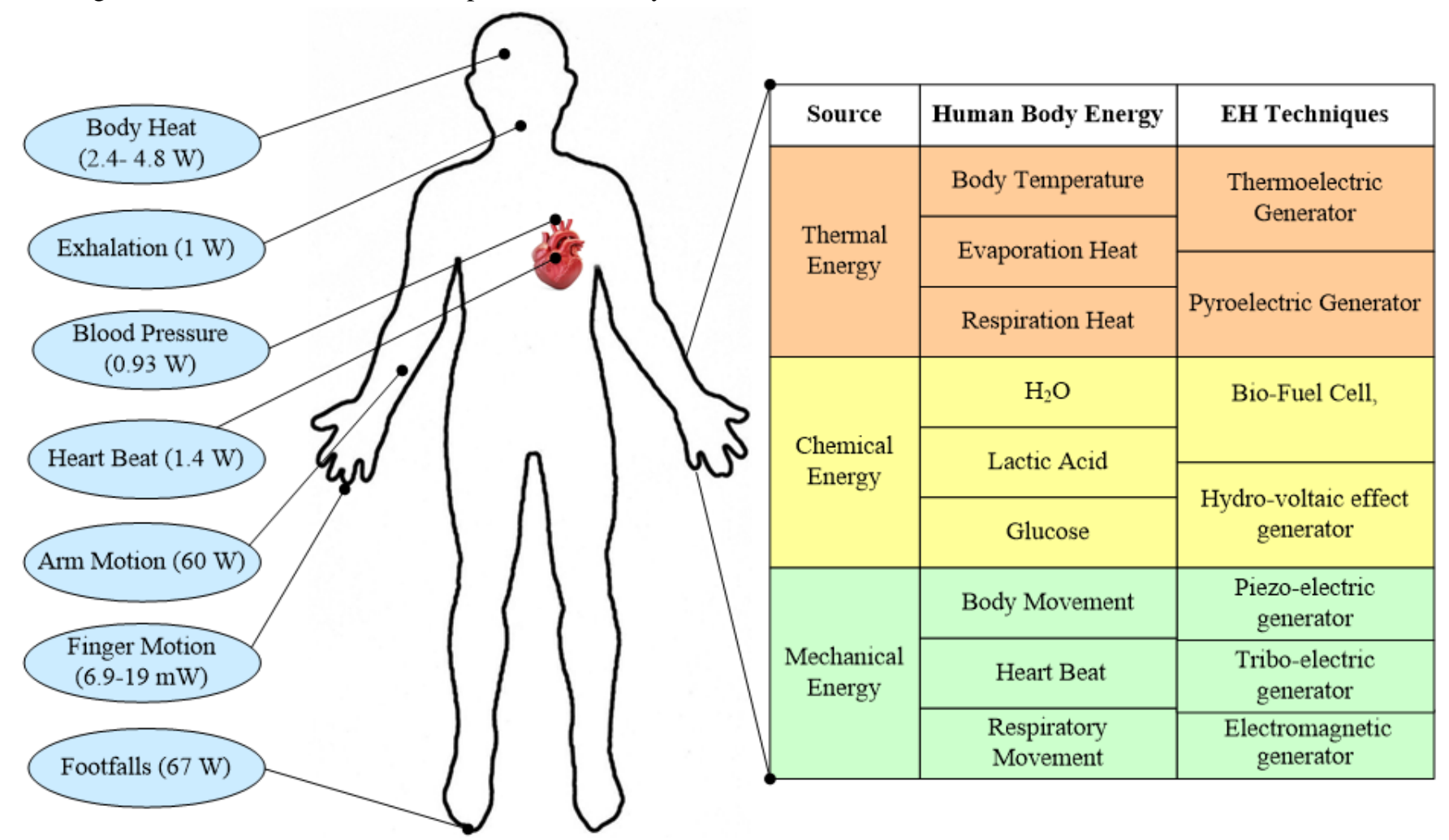

Fig. 1. Energy Distribution in Human Body and its Energy Harvesting Techniques [7] 


\section{WORKING PRINCIPLES OF BIOMECHANICAL ENERGY HARVESTING}

The biomechanical energy is harvested from the human body using the most common methods like Piezoelectric, Triboelectric, and Electromagnetic. The principles of the piezoelectric effect are used for the operation of piezoelectric materials. Many researchers can use the Piezoelectric Harvesting in humans to energize intelligent wearable devices like a smart watch, smart skin, smart shoes, portable and implantable devices. The most recent and advanced technologies are incorporated in Triboelectric energy harvesting. This approach uses the triboelectrification phenomenon for charging (electrical) the materials using frictional contact. The electricity is generated based on electrically charged materials with relative displacement. The recent human body-based energy harvesting is performed using triboelectric energy harvesting because of high power outputs, performance, and nanoscale designs. Electromagnetic Energy harvesting is working based on electromagnetic induction (Faraday's law). Faradays, law states that the electric current is induced based on the property of the materials used through a magnetic field. There are many kinds of research conducted based on electromagnetic energy harvesting for self-operated wearable electronic devices. However, these on electromagnetic energy harvesting system lacks with miniaturized designs and device size. The working principles, and recent work, and applications are discussed in the following sections.

\section{A. Piezoelectric Energy Harvesting (PEEH)}

Piezoelectric Energy harvesting (PEEH) is a widely used harvesting method due to its simple design and accessible materials usage. Piezoelectricity is observed macroscopically to produce electricity through molecular phenomena when some substances (i.e., piezoelectric devices) are deformed. The linear-piezoelectric effect (direct and indirect) [10-11] is represented in (1) and (2) as follows:

$E_{D}=c \sigma+\in . E F_{i}$

$\varepsilon=s \sigma+c E F_{i}$

Where $\sigma$ and $\varepsilon$ are stress and strain constants; The ED is electric displacement, and $\mathrm{EF}_{\mathrm{i}}$ is electric field intensity; $\mathrm{s}$ is elastic compliance, $\in$ is permittivity, and c is Piezoelectric (PE) co-efficient.

The direct effect of PE is defined in (1), which converts the mechanical energy into electric energy using $\mathrm{PE}$ materials. The electrical energy is produced by applying the mechanical force using crystalline materials (Quartz, salt, titanate) directly. In contrast, the indirect (converse) effect of $\mathrm{PE}$ is defined in (2), which converts the electric energy into mechanical energy using PE materials. When the electric field is applied to these materials, which produces mechanical energy in deformation. The Linear Piezoelectric energy harvester produces the narrow bandwidth for particular object frequencies, limiting the harvester compared to other non-harmonic systems. The nonlinear piezoelectric Energy harvesting system based on vibration overcomes the drawbacks of Linear PEEH [12].
The Piezo electrical materials [13-14] are categorized into two parts: Piezoceramics and Piezopolymers (PVDT). The Piezopolymers materials are easy to use and flexible but provide low-energy transformation rates and low electromechanical co-efficient. At the same time, Piezoceramics are complex and easily breakable. These materials provide high-energy transformation rates and significant electromechanical coefficients. The Piezoceramic materials like PZT8, PZT-5H, and Piezopolymers- (PVDT) have their material properties. The PEEH is classified further based on principles and materials used: Piezotronics mode, longitudinal mode (d31), and Transverse mode (d33). The PEEH limitation factor is that the energy harnessing will be significantly less; the energy produced only during mechanical stress and harnessing is very tough as the piezoelectric materials are very toxic. The predicted research of many researchers has suggested that Piezoelectricity's applicability can be effective in Nanotechnology.

\section{B. Triboelectric Energy Harvesting (TEEH)}

The TEEH works based on the principles of triboelectrification. The Energy transfer of the triboelectric effect works based on dissimilar polarities of two materials that can contact other two materials, which creates the opposite charges on each side of the electrical surface. The concept of triboelectricity was initiated long back, where electrostatic charges are generated when two materials are rubbed together. The materials of TEEH are placed in a single column, and one of the triboelectric series placed in the bottom gets a negative charge when they are rubbed with the above one. The primary Voltage $\left(\mathrm{V}_{\mathrm{e}}\right)$ between two metal electrodes for the given charge accumulation $\left(\mathrm{Q}_{\mathrm{c}}\right)$ on the surface is represented in (3) as follows [30]:

$V_{e}=\frac{Q_{c} D_{e}}{\epsilon_{0} S_{e}}$

The External electrodes produce the Induced current $\left(\mathrm{I}_{\mathrm{e}}\right)$ and are represented in the (4):

$I_{e}=C_{e} \frac{\partial V_{e}}{\partial t}+V_{e} \frac{\partial C_{e}}{\partial t}$

Where $\mathrm{S}_{\mathrm{e}}, \mathrm{D}_{\mathrm{e}}, \epsilon_{0}, \mathrm{C}_{\mathrm{e}}$ are metal electrode surface area, interlayer distance, Vacuum permittivity, and Capacitance of the Triboelectric system.

The triboelectric materials produce positive or negative charges based on the contact electricity in the human body. The Skin (Human body) gains the top similarity to lose a positive electron, and in contrast, the Fluoropolymer tends to gain negative electrons at a higher rate. The four different TEEH working operations are classified based on types of similar motion between two triboelectric materials and electrode position. The TEEH process operations include Single Electrode mode, sliding contact mode, Freestanding Mode, and Vertical contact separation mode. These TEEH are the most used technology in recent times for human motions. Most of these works are implemented on wearable and implantable electronic devices. 


\section{Electromagnetic Energy Harvesting (EMEH)}

The Electromagnetic Energy Harvesters (EMEH) work is based on faraday's law with electromagnetic induction [47]. When both ends of the coils produce a nominal difference via a magnetic field. The Voltage (V) used across the coil is directly proportional to the rate of change of magnetic flux. The Voltage and magnetic flux $(\varphi)$ are expressed in equation (5) as follows [67]:

$V=N \frac{d \varphi}{d t}$ And $\varphi=\sum_{i=1}^{N} \int_{A} B \cdot d A$

Where ' $\mathrm{N}$ ' represents the coil's number of turns, the overall area of the $\mathrm{i}^{\text {th }}$ coil is ' $\mathrm{A}$ ', and the magnetic field is denoted by ' $\mathrm{B}$ '. The electricity is generated in EMEH using a magnet and conductor (relative motion of the coil). The EMEH is working with two modes of operation. (i) The coil area is changed while placing the coil perpendicular to the magnetic field. magnetic field's intensity is always uniform and constant across the conductor. (ii) The coil was moving parallel with the magnetic field's direction and followed by the magnetic field's intensity, constantly changing with the uniform coil's area. The three different types of EHEH working operations are classified based on mechanical motion. The EMEH working operations include oscillatory EH, rotational $\mathrm{EH}$, and hybrid forms of $\mathrm{EH}$.

\section{ReCENT PRogress in Biomechanical ENERgy HARVESTING SYSTEM USING HUMAN BODY}

The recent progress in biomechanical energy harvesting techniques is discussed in this section.

\section{A. Piezoelectric Energy Harvesting (PEEH)}

The recent works on the PE-based energy harvesters are discussed for human motion in the section. Wang et al. [15] present the study of the Piezotronics and Piezophotochromic nanoarchitectures in addition to nanogenerators of Triboelectric, piezoelectric, and blue energy. The Usage and its application fields are analyzed for the same.

Granstrom et al. [16] present the energy harvesting of backpack instruction for piezoelectric shoulder straps. The design uses polyvinylidene fluoride (PVDF) materials for the backpack and consumes $45.6 \mathrm{~mW}$ power while performing the simulation. The thickness of the shoulder straps is $52 \mu \mathrm{m}$ using PVDF material.

Calio et al. [17] discuss the study of Piezoelectricity and its material choice in detail with working operations. Pillatsch et al. [18] discuss the human motion energy harvester using wireless power transfer. The design uses the frequency upconversion method for piezoelectric energy harvesting. Fan et al. [19] present a collection of energy from human lower limbs using the PEEH approach. The walking speed increases from
$2 \mathrm{~km} / \mathrm{h}$ to $8 \mathrm{~km} / \mathrm{h}$ when the PEEH's output voltage increases. The work also states that up-conversion is possible by using ultra-low frequencies of human motions. Wang et al. [20] discuss the nonlinear PEEH from human motion and analyze the frequency method's effectiveness to check the optimal resistance. The work is demonstrated on the Treadmill by placing the EH device on the Human leg. The motion speed is varying based on the resistance value changes. The EH utilizes $30.55 \mu \mathrm{W}$ power at the $7 \mathrm{~km} / \mathrm{h}$ speed.

Wang et al. [21] present the PEEH using a Bionic Single electrode electronic skin unit. The Single-electrode piezoelectric nanogenerators (SPENG) is designed using PVDF non-fibers to maintain steady-state sensing. The SPENG overcome the drawbacks of the Single-electrode triboelectric nanogenerators (STENG) for electronic skins. Kim et al. [22] present the multi-directional PEEH with flexibility for Human motion. The PEEH design incorporates the polydimethylsiloxane (PDMS) bump with PVDF material to maintain flexibility and access multidimensional inputs from human motion. Kakihara et al. [23] investigate the PEEH from walking using PVDF materials. The work produces less than $50 \mu \mathrm{W}$ power with a kinetic energy of $500 \mu \mathrm{J} / \mathrm{s}$. Guido et al. [24] present the Aluminum Nitride (AIN) thin-film-based PEEH from human motion for the skin. The work is validated using the Finite Element Method (FEM) for electrical energy and mechanical deformation. Wu et al. [25] discuss the PEbased spring pendulum oscillator for low-frequency vibration harvesting systems with multi-directional features, represented in Fig. 2(a). The device consumes the $13.29 \mathrm{~mW}$ power at $2.03 \mathrm{~Hz}$ frequency, and it is applicable for low-power wearable applications. Qian et al. [26] present the PE footwear-based $\mathrm{EH}$, which provides the average power of $9 \mathrm{~mW} / \mathrm{shoe}$ with a walking speed of $4.8 \mathrm{~km} / \mathrm{h}$. The footwear EH consumes $28 \mathrm{~mW}$ with an open-circuit voltage of 20V. The polydopamine (PDA) based PE pressure sensor is designed by Yang et al. [72], and it is represented in Fig. 2(b). The PDA uses barium titanate (BTO) with PVDF composite film for human motion monitoring by using 9.3V output Voltage.

The applications of the PEEH using human motion are represented in Fig. 3. Smart Shoes, smart Textile, smart skin, Biomonitoring, and Implantable devices are the major applications of PEEH using human motions. Fig. 3(a) shows the PE-based shoulder straps [16] with backpack instrumentation for energy harvesting. The user (Human) and backpack are closely packed to improve the performance and harvest the energy. The PEEH module is fixed on the shoes [19] and analyzes the performance while running on Treadmill. The energy harvested results are displayed in the oscilloscope are represented in Fig. 3(b). The PENG-based electronic skin [21] is designed using a single electrode method shown in Fig. 3(c). 

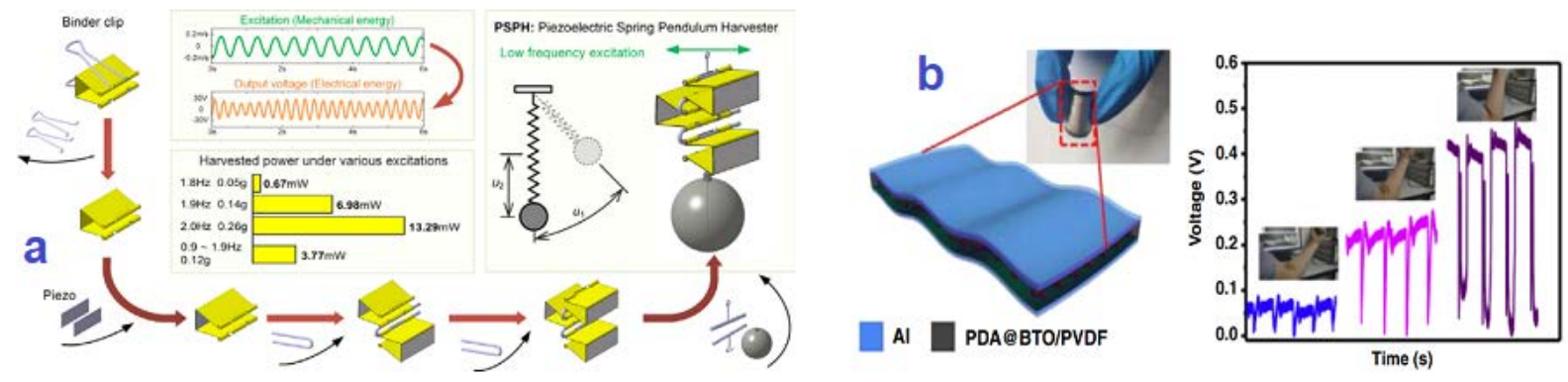

Fig. 2. PEEH Working Operations: (a) PE-based Spring Pendulum Harvester [25], (b) PDA based PE Pressure Sensor [72].
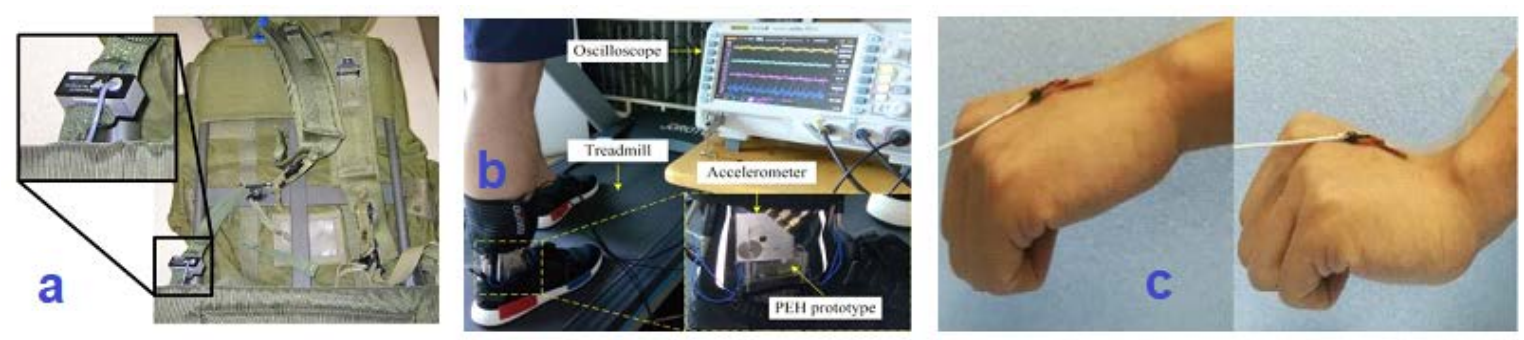

Fig. 3. Applications of PHEH: (a) Shoulder Straps [16], (b) Smart Shoes [19], (c) E-skin [21].

\section{B. Triboelectric Energy Harvesting (TEEH)}

The recent works on the TE-based energy harvesters are discussed for human motion in this section. Wang et al. [27] present the triboelectric-based $\mathrm{EH}$ to sustain the power in portable electronic devices. The arch-shape-based TE nanogenerator (TENG) is designed to direct electricity between the thin metal film and polymer thin film. The TENG is demonstrated on a Mobile phone to charge the Lithium battery is shown in Fig. 4(a). In addition, TENG [28] is used as a selfpower sensor and provides better energy efficiency than the TENG [27]. The Sliding based TENG is designed based on Inplane charging separation (IPCS) method by Wang et al. [29]. The IPCS process is done using a relative sliding operation using two surface contacts. Fan et al. [30] present the Flexible nanogenerators for self-power electronics and also for EH. The hybrid nanogenerator mechanism is designed by using both $\mathrm{PE}$ nanogenerators (PENG) and TENG. Hybrid nanogenerators are used in many self-power devices and also for energy harvesting. In 8addition, that Hybrid generators using both PENG and TENG by Song et al. [31]. The work illustrated both wearable bio devices and bio-electronics. Wang et al. [32] present the TENG for EH from human motion or moving objects in non-contact and contact modes. The designed TENG supports freestanding features in the TE layer, which moves to and fro using external mechanical energy. Huang et al. [33] discuss the all-fiber TENG, which has PVDF PE nanofibers for human walking and wearable devices. The human walking results are analyzed, and it consumes $2.1 \mathrm{~mW}$ power with $210 \mathrm{~V}$ maximum output voltage. Tang et al. [34] present the Highperformance TENG using the Liquid Metal electrode method, which provides instantaneous energy conversion with an efficiency of $70.6 \%$, five times better than the Solid thin filmbased electrode method. Ha et al. [35] present the TE-based generators and sensors for wearable electronic devices with self-powered features. The generators like Planar, Micropatterned TEG, Multi-layered TEG, Rotary-type TEG are designed for Wearable devices like smart glass, watch=, smart band, smart ring, and smart lens. Lin et al. [36] present the TENG-based body sensor network (BAN) system is used in heart-rate monitoring system of human body with the selfpower mechanism. Human walking is demonstrated, which consumes the power of $2.28 \mathrm{~mW}$ with a conversion efficiency of $57.0 \%$. Shen et al. [37] present the TENG for high Performance-based Bio-mechanical EH system with a Humidity resisting feature. This work is demonstrated on human movements for wearable electronics. The device works at an output voltage of $345 \mathrm{~V}$, current of $28 \mu \mathrm{A}$ with $55 \%$ relative humidity.

Yu et al. [38] present the TEGN for textiles as power cloths using the Core-shell-Yarn method. The Textile based TENG work provides flexible, fashionable, comfortable features and is further used for large-scale textile manufacturing. Chen et al. [39] present the TENG using Ultra-thin Flexible Single electrode method for Mechanical EH and instantaneous force sensing. Zhang et al. [40] discuss the stretchable electrodebased TENG using Air cushion features for human motions. The work outputs are validated with Lighting LEDs, power supplies for the calculator, and a digital thermometer. Ding et al. [41] explain the operation of TENG with a large scale for human sleep monitoring. The device supports a flexible sensor with self-powered features for monitoring human sleep with high-power output and high sensitivity. Ning et al. [42] discuss the Single-electrode based TENG with Textile structure (TS) for Wearable electronics with self-powered features. The design works with a current of $22 \mu \mathrm{A}$ and consumes the output voltage of $1050 \mathrm{~V}$.

Song et al. [43] discuss the Smart bracelet-based TENG with high efficiency for portable electronics with a selfcharging feature. The device obtains the peak voltage of 305V and consumes $300.4 \mu \mathrm{W}$ power with $69.3 \%$ energy efficiency. Xia et al. [44] present the milk-based TENG for EH using Human body motions. The work consumes $4.67 \mathrm{~mW}$ of power, $392 \mathrm{~V}$ of peak voltage with a power density of $583.75 \mu \mathrm{W} / \mathrm{cm} 2$ 
for food quality detection using the human arm. Wang et al. [45] present the skin-based TENG using transparent polyionic materials for human motion EH with highly flexible features. The design works consume the power of $1.12 \mathrm{~mW}$ with an open-circuit voltage of $70 \mathrm{~V}$. Xiong et al. [46] discuss the Textile based TENG for Biomechanical EH with black phosphorous materials. The Textile-based TENG with Washable skin touched-actuated features is demonstrated, providing the $60 \mathrm{~V}$ low Voltage from Cloth/skin and is used from human motion. Tian et al. [71] designed the TENG-based electrical stimulator, which supports osteoblasts differentiation and proliferation. The design is used for self-powered implantable devices and is shown in Fig. 4(b).

The applications of the TEEH using human motion are represented in Fig. 5. Biomedical monitoring, Smart footwear, smart clothes, smart watch, smart skin are the major applications of TEEH using human motions. The human walking controlled wearable TENG [33] is designed using electrospun PVDF non-fibers, shown in Fig. 5(a).

The Single electrode-based TENG is designed for wearable electronic devices, especially washable cloths [42] with selfpower features in Fig. 5(b). The self-powered smart bracelet [43] is designed using freestanding TENG, and it's shown in Fig. 5(c). Finally, the transparent polyionic skin [45] based TENG is designed for human motion energy harvesting with flexible features represented in Fig. 5(d).

\section{Electromagnetic Energy Harvesting (EMEH)}

The recent works on the EM-based energy harvesters are discussed for human motion in this section. Dai et al. [48] discuss the electromagnetic generator (EMG) with hipmounted features for EH using human motions. The amount of kinetic energy from walking and running is converted into electrical energy. The design consumes the open-circuit Voltage of $1 \mathrm{~V}$ by consuming the $284 \mu \mathrm{W}$ of power. Luciano et al. [49] present the EMEH for total knee prosthesis (TKP) of humans; the TKP works with internal loads to improve longer durations. The design consumes $1.7 \mathrm{mw}$ power for every $7.6 \mathrm{~s}$ of walking by utilizing the $2 \mathrm{~V}$ of Voltage. Zhang et al. [50] discuss the EMEH using coil arrays and magnets with magnetic springs for the energy generation from human motions. Dinuloyic et al. [51] present the EMEH system with rotational features. The design has multi-layer planar coils with magnets and a mechanical system for movement. The design consumes an $800 \mathrm{~mA}$ short circuit current with an open-circuit voltage of 2V. Quan et al. [52] present the EM-TENG with hybrid features for a self-powered electronic wristwatch. The watch is operated continuously for 456 seconds by charging the capacitor of $100 \mu \mathrm{F}$ in 39seconds. Bendame et al. [53] discuss the springless vibration EH system using EM impact, which supports wide bandwidth with low-level vibrations. Niroomand et al. [54] discuss the EM-microgenerator with rotary features for EH using human motions. The design uses the open-circuit Voltage of $0.3 \mathrm{~V}$ and consumes the $284 \mu \mathrm{W}$ of power by performing normal walking.

Geister et al. [55] present the nonlinear-based EMEH for wearable sensors with scaling effects. The design obtains the $150 \mathrm{mV}$ of Root means square (RMS) output voltage and consumes the $530 \mu \mathrm{W}$ of load power. Brunner et al. [56] discuss the EMEH system for the upper extremity of the human body. The EM-linear Generator for Upper Limb is represented in Fig. 6(a). The module generates the gyrating mass, which consumes the average power of $50 \mu \mathrm{W}$ with an output power of $2.2 \mathrm{mw}$. Halim et al. [57-58] present the EMEH using the rotational eccentric mass method, as shown in Fig. 6(b). The design uses the Swing arm motion for energy generation by consuming $55 \mu \mathrm{W}$ of average power. In addition to that, the other EMEH using sprung eccentric rotor is designed from human motion with pseudo-walking. This works $61.3 \mu \mathrm{W}$ power with $1 \mathrm{~Hz}$ frequency, which is greater than the upsprung rotor part. Zhang et al. [59] present EMEH using the circular Halbach array Method from bearing motion. The design utilizes the rotational speed is varied from 600-1000rpm by consumes the average power of $50.8-131.1 \mathrm{~mW}$ with a harvested output voltage of $2.79 \mathrm{~V}-4.59 \mathrm{~V}$. Fan et al. [60] present the EMEH using nonlinear two-degree freedom features for human body motions. The works are applicable for Ultra-low-frequency vibrations and consume $2.58 \mathrm{~mW}$ of power with an output voltage of 0 to $5 \mathrm{~V}$.

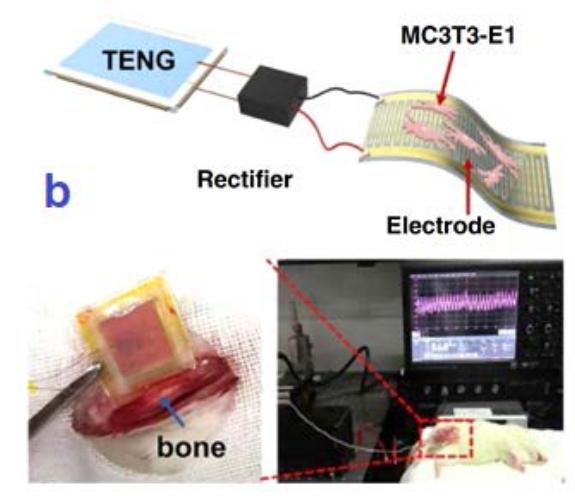

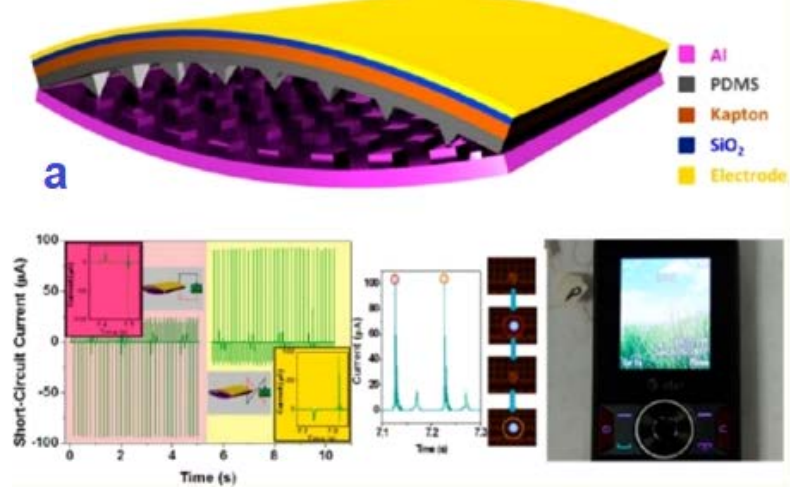

Fig. 4. TEEH Working Operations: (a) Arch Shaped TENG and its Results [27], (b) TENG based Implantable Stimulator [71]. 

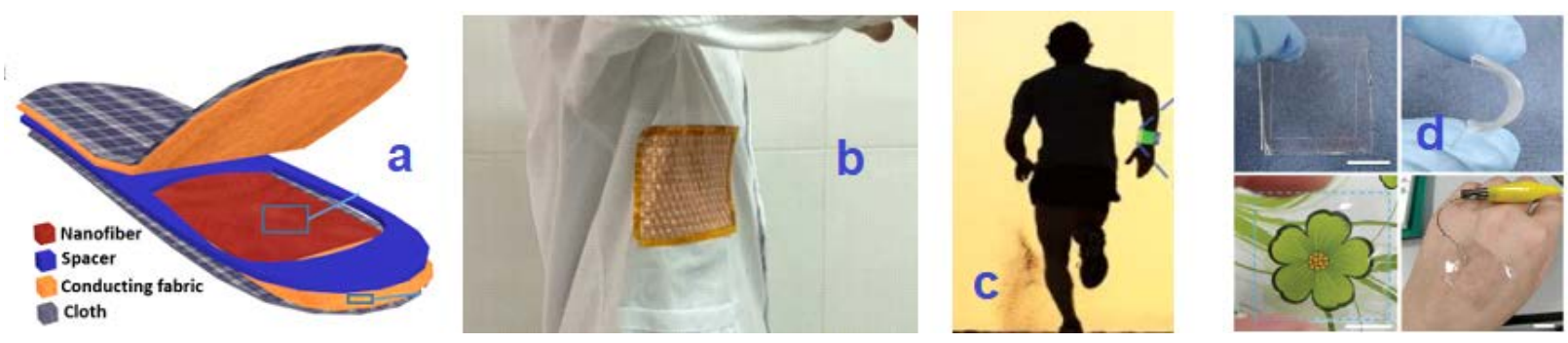

Fig. 5. Applications of TEEH: (a) Smart Shoes [33], (b) Smart Cloths [42], (c) Smart Watch [43], (d) Smart Skin [45].
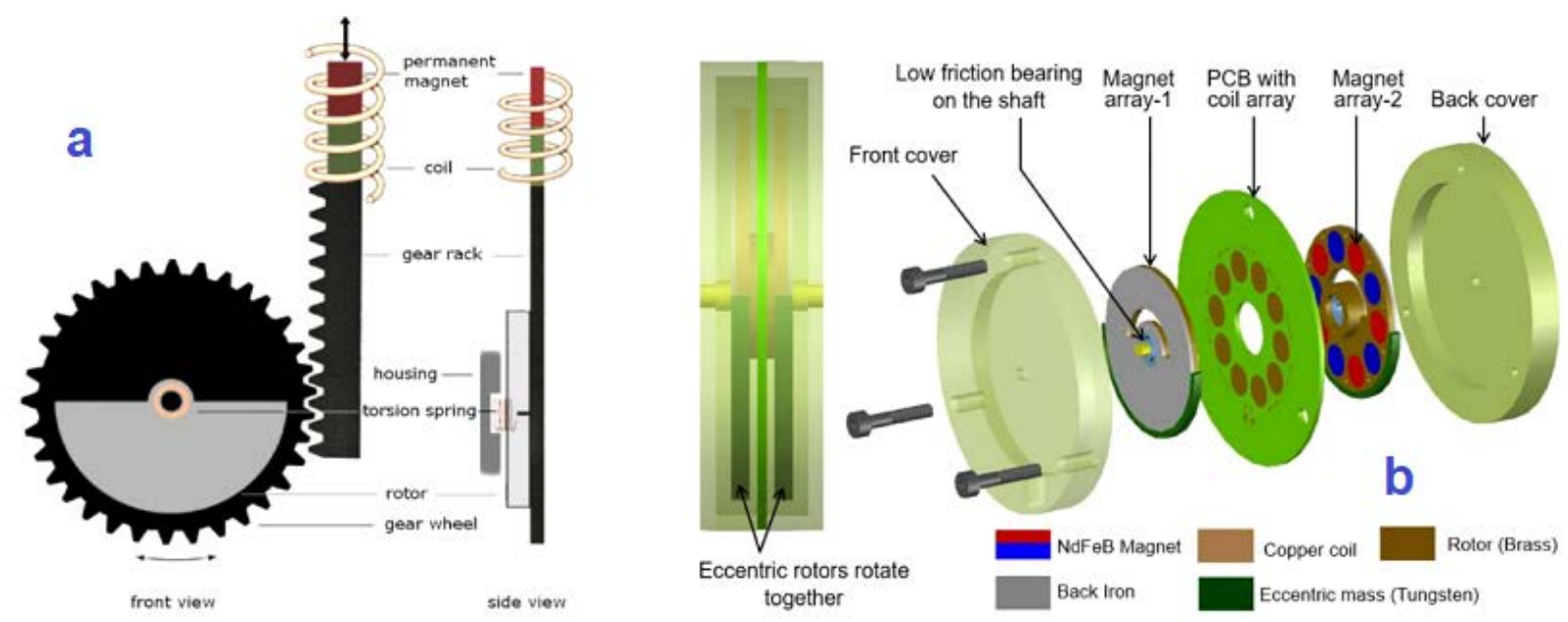

Fig. 6. EMEH Working Operations: (a) EM-Linear Generator for Upper Limb [56], (b) Rotational EMEH for Swing-Arm Motion [57].
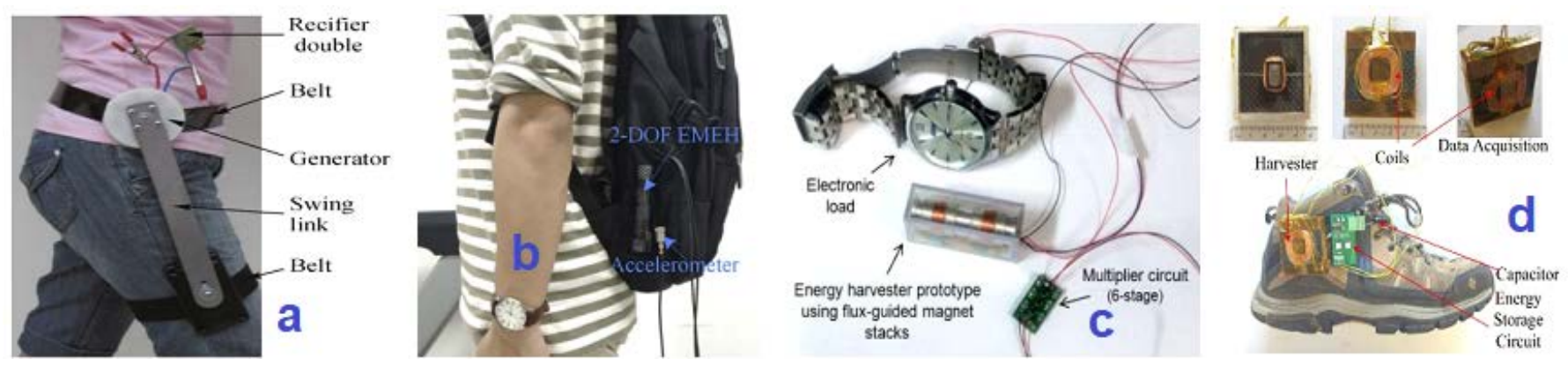

Fig. 7. Applications of EMEH: (a) Hip Mounted Leg [48], (b) Smart Arms [60], (c) Smart Wrist Watch [68], (d) Smart Shoes [69].

The applications of the EMEH using human motion are represented in Fig. 7. Biomedical monitoring, Smart skin, smart clothes, smartwatch, smart shoes are the major applications of EMEH using human motions. The Hip mounted leg [48] using an EM generator is designed to harvest the energy, represented in Fig. 7(a). Fig. 7(b) shows the twodegree of freedom (2-DOF) based EMEH is designed for human arm with ultra-low frequency vibrations. The smart wristwatch [68] is designed using flux-guided magnet stacks in EMEH and is shown in Fig. 7(c). Fig. 7(d) shows the Threedegree of freedom (3-DOF) based human motion EMEH for smart shoes [69] using ferrofluid.

The human body produces the energy harvested using three different techniques like PEEH, TEEH, and EMEH. These EH's produces energy using specific human body parts and energy resource characteristics. The application (Smaller or big) requirements are categorized based on the amount of energy harvested. Many strategies are applied to produce the energy with the help of a human body for implantable and wearable devices [63-66]. The Summary of Wearable and implantable devices on the Human Body is tabulated in Table I.

\section{Comparison between Different Energy Harvesters}

The review of the recent works of bio-mechanical energy harvester systems on the Human body produces different applications, performance metrics, advantages, and limitations. The advantages and limitations of the different Energy harvesters on the Human body are summarized in Table II. The parameters like Power density usage, EH from human body parts, Usage of different methods, and recent development are summarized in Table III. Finally, the different energy harvesters produce the output power (W) and output voltages (V) using different human body parts and are summarized in Table IV. 
TABLE I. SUMmary of WEARABLE AND IMPLANTABLE DEVICE ON HUMAN Body

\begin{tabular}{|l|l|l|l|}
\hline Human Body Part & Wearable Device & Human Body Part & Implantable Devices \\
\hline Legs & Smart Power shoes & Brain & Deep Brain Stimulator \\
\hline Legs & Socks: sweat analyzer & Muscle & Muscle stimulator \\
\hline Body & Microneedle: Blood analyzer & Bladder & Bladder pressure sensor \\
\hline Body & E-skin: Man-machine interface & Heart & Symbiotic Cardiac Pacemaker \\
\hline Body & Clothing: Smart Fabrics & Aorta & Blood pressure sensor \\
\hline Hand & Wristband: Pulse sensor & Stomach & Vagus Pressure Stimulator \\
\hline
\end{tabular}

TABLE II. AdVANTAgES ANd Limitations of DifFERENT ENERgy HaRVESTERs ON THE HuMAN Body

\begin{tabular}{|c|c|c|}
\hline Methods & Advantages & Limitations \\
\hline Piezoelectric EH & $\begin{array}{l}\text { Higher Power density; } \\
\text { Produce Higher open circuit Voltages, Flexible and } \\
\text { smaller in size, } \\
\text { Highly sensitive to the applied strain } \\
\text { Used for both Sensor technology and EH }\end{array}$ & $\begin{array}{l}\text { Produces lower current and high impedance, } \\
\text { Less-efficient for lower-frequency designs, } \\
\text { Need of unique materials, } \\
\text { High-performance PE materials are hard but easily breakable } \\
\text { Requires higher cost to produces Higher energy output, }\end{array}$ \\
\hline Triboelectric EH & $\begin{array}{l}\text { Easy Fabrication, } \\
\text { Low-cost, flexible, scalable, } \\
\text { wide selection of materials, } \\
\text { Produce very high-power density, } \\
\text { Produce Higher output Voltages, } \\
\text { Free from maintenance cost }\end{array}$ & $\begin{array}{l}\text { The surface is rough and clean, } \\
\text { Needs High voltage insulation, } \\
\text { Initial understanding is limited, } \\
\text { produces lower current density, } \\
\text { Charge induction and polarity is depending on the material used } \\
\text { Ambient Humidity causes the output performance }\end{array}$ \\
\hline $\begin{array}{l}\text { Electromagnetic } \\
\text { EH }\end{array}$ & $\begin{array}{l}\text { Produces higher output current } \\
\text { Small size, Voltage requirement to run the } \\
\text { operations is not required }\end{array}$ & $\begin{array}{l}\text { Produces the Lower output voltage, } \\
\text { Less flexible, } \\
\text { challenging to make smaller-scale magnet and coils, } \\
\text { Integration with microsystem is complex, } \\
\text { Caused by Vibrations (Amplitude, frequency) and damping factors }\end{array}$ \\
\hline
\end{tabular}

TABLE III. DIFFERENT PARAMETERS SUMmARy OF DIFFERENT ENERgy HARVESTERS ON THE HuMAN BOdY

\begin{tabular}{|c|c|c|c|}
\hline Parameters & Piezoelectric EH & Triboelectric EH & Electromagnetic EH \\
\hline Power Density & $\begin{array}{l}\text { Wearables: } 37 \mathrm{~mW} / \mathrm{cm}^{2} \\
\text { Implantables: } 1.2 \mu \mathrm{m} / \mathrm{cm}^{2}\end{array}$ & Wearables: $-3 \mathrm{~mW} / \mathrm{cm}^{2}$ & $\begin{array}{l}\text { Wearables: } 507 \mu \mathrm{W} / \mathrm{cm}^{2} \\
\text { Implantables: }-4 \mu \mathrm{W} / \mathrm{cm}^{2}\end{array}$ \\
\hline $\begin{array}{l}\text { Human Body } \\
\text { Parts }\end{array}$ & $\begin{array}{l}\text { Shoulder straps [16] } \\
\text { Lower Limbs [19] } \\
\text { Human Leg [20] [23] } \\
\text { Electronic Skin [21] [24] } \\
\text { Walking [23] } \\
\text { Footwear [26] }\end{array}$ & $\begin{array}{l}\text { Shoe [33] } \\
\text { Cloth [38] [42] } \\
\text { Wrist Bracelet[43], Watch [61] } \\
\text { Arm [44] } \\
\text { Skin [45] } \\
\text { Cloth/Skin [46] }\end{array}$ & $\begin{array}{l}\text { Hip mounted [48] } \\
\text { Knees [ 49] } \\
\text { Watch [52] [68] } \\
\text { Walking [54] [58][62] } \\
\text { Arm [60], Shoes [69] } \\
\text { Leg and Arm [70] }\end{array}$ \\
\hline Methods & $\begin{array}{l}\text { Circuit Usage [17] } \\
\text { Frequency up-conversion [18] } \\
\text { Non-linearity [20] } \\
\text { Spring pendulum oscillator [25] }\end{array}$ & $\begin{array}{l}\text { In-plane charging separation method [29] } \\
\text { Liquid metal electrode [34] } \\
\text { Core-shell structure method [38] } \\
\text { Ultrathin flexible single-electrode [39] } \\
\text { Air-cushion method [40] }\end{array}$ & $\begin{array}{l}\text { Spring-less system [53] } \\
\text { Spring clockwork Method [50] [56] } \\
\text { Sprung eccentric rotor [58] } \\
\text { Circular Halbach array Method [59] } \\
\text { Non-linearity [55] [60] } \\
\text { Frequency up-conversion [70] }\end{array}$ \\
\hline $\begin{array}{l}\text { Recent } \\
\text { Developments }\end{array}$ & $\begin{array}{l}\text { Flexible and stretchable PENG, } \\
\text { Nanocrystals Usage in PENG }\end{array}$ & $\begin{array}{l}\text { Nanocrystals Usage in TBNG, Flexible } \\
\text { Generator, Cantilever-based TEG, Triboelectric } \\
\text { Fabric [33] }\end{array}$ & Flexible Generator \\
\hline
\end{tabular}


TABLE IV. Performance Metrics Summary of DifFERENT ENERgy HARVESTERS ON THE Human Body

\begin{tabular}{|c|c|c|c|}
\hline Methods & Human body Part & Output Power (mW) & Output Voltage (V) \\
\hline Piezoelectric EH & $\begin{array}{l}\text { Shoulder straps [16] } \\
\text { Human Leg [20] } \\
\text { Electronic Skin [24] } \\
\text { Footwear [26] }\end{array}$ & $\begin{array}{l}45.6 \\
30.55 \\
0.0002 \\
28\end{array}$ & $\begin{array}{l}\text { NA } \\
\text { NA } \\
0.7 \\
20\end{array}$ \\
\hline Triboelectric EH & $\begin{array}{l}\text { Shoe [33] } \\
\text { Heart rate monitor [36] } \\
\text { Cloth [42] } \\
\text { Wrist Bracelet [43] } \\
\text { Arm [44] } \\
\text { Skin [45] } \\
\text { Cloth/skin [46] }\end{array}$ & $\begin{array}{l}2.1 \\
2.28 \\
\text { NA } \\
0.3004 \\
4.67 \\
1.12 \\
\text { NA }\end{array}$ & $\begin{array}{l}210 \\
\text { NA } \\
1050 \\
305 \\
392 \\
70 \\
60\end{array}$ \\
\hline Electromagnetic EH & $\begin{array}{l}\text { Hip mounted [48] } \\
\text { Knees [49] } \\
\text { Walking [54] } \\
\text { Upper limb [56] } \\
\text { Arm [60] } \\
\text { Shoes [69] } \\
\text { Wrist Watch [68] } \\
\text { Leg and Arm [70] }\end{array}$ & $\begin{array}{l}0.284 \\
1.7 \\
0.416 \\
2.2 \\
2.58 \\
2.28 \\
0.203 \\
0.5\end{array}$ & $\begin{array}{l}2.5 \\
2 \\
0.3 \\
\text { NA } \\
0 \text { to } 5 \\
0.5 \\
1.12 \\
0.24\end{array}$ \\
\hline
\end{tabular}

\section{CHALlENGES AND SOLUTIONS}

The critical challenges for different bio-mechanical energy harvesters from human motion are addressed from the review observations.

- The PEEH produces very ultra-low frequencies around $1 \mathrm{~Hz}$ from human motion; however, The PEEH operated with very high bandwidth, compensating human motions. In addition, the Ceramic based piezoelectric materials are rigid and stiff but easily breakable. Therefore, the Linear PEEHs are challenging to extract the average power from human complex or multidimensional motions.

- The TEEH faces mechanical impact issues, which cause many problems (Output performance degradation, Reduction in life span, and safety hazards) and device failure. The moisture and dust particles cause the output

- Performance in the TEEH surface. So, there are chances to cause reliability, durability, and robustness issues. Humidity always affects the performance of the system. Washability issues, electrodes are not flexible in TENG operation,

- The EMEH system naturally uses low-frequency-based devices, which is challenging to produce the maximum output power. In contrast, ultra-lower frequencies are produced from human motion and unsuitable for producing the energy from human body or motion. Integration is a big challenge in EMEH and also tricky to miniaturizing the system. The size and height of the EMEH are enormous. The EMEH system is not flexible in Nature.

The possible solutions for the challenges and issues of the different biomechanical energy harvesters from human motion are addressed.

- The Usage of microfabrication methods like MEMSbased PEEH leads to produces in further progress the
Nano PEEH to improve integration issues and output performance. In addition, the Cantilever beam designs are incorporated in PEEH to improve the flexibility in multidimensional motions.

- The Multi-layered encapsulation technique improves the reliability and durability problems irrespective of the environmental conditions. In addition, the TE material abrasion is reduced by using liquid-solid contact in TEEH, which also improves the humidity issues.

- The low-frequency-based springless oscillators are used in EMEH to improve the human motion energy harvesting from the ultra-low frequency range. Frequency-up conversion methods are adopted to improve the output power in the EMEH system (Spring-mass-damper system). Most of the researchers are using hybrid generators like EMEH with TEEH to reduce the size (miniaturization) and also improve the power output.

\section{CONCLUSION AND Future SCOPE}

In this manuscript, a detailed review of biomechanical energy harvesting from human motion is discussed. These biomechanical energy harvesters are mainly used in wearable and implantable electronic devices like smart shoes, smartwatches, smart skins, smart clothes, and many more. Harvesting the bi-mechanical energy from human motions is a challenging task. Human movements like walking, arm movement, muscle exercise produces very low frequencies (<10 Hz.). The Bio-mechanical energy harvesters are categorized into three parts PEEH, TEEH, and EMEH. The working principles of the three above energy harvesters are discussed with mathematical equations. The recent progress and development of the Biomechanical energy harvesters are discussing in detail with applications. The advantages and limitations of the PEEH, TEEH, and EMEH are summarized. The performance metrics, recent development, and methods are summarized in detail with Tabulation. Lastly, the Biomechanical energy harvesting system challenges and possible 
solutions are addressed. In the future, incorporate the biomechanical energy harvesters into the closed loop-bio electronic system with self-power features.

\section{REFERENCES}

[1] I. Chopra, "Review of the state of the art of smart structures and integrated systems," AIAA Journal, vol. 40, no. 11, pp.2145-2187, 2002.

[2] A. Proto, L. Peter, M. Cerny, M. Penhaker, D. Bibbo, S. Conforto, and M. Schmid, "Human Body Energy Harvesting Solutions for Wearable Technologies," In IEEE 20th International Conference on e-Health Networking, Applications and Services (Healthcom), pp. 1-5, 2018.

[3] S. Roundy, and S. T-McKinstry, "Materials and approaches for on-body energy harvesting," MRS Bulletin, vol. 43, no. 3, pp. 206-213, 2018.

[4] H. Shi, Z. Liu, and X. Mei, "Overview of human walking induced energy harvesting technologies and its possibility for walking robotics," Energies, vol. 13, no. 1, pp. 86, 2020.

[5] G. Rebel, F. Estevez, P. Gloesekoetter, and J. M. C-Secilla, "Energy harvesting on human bodies," In Smart Health, pp. 125-159, 2015.

[6] H. Wang, A. Jasim, and X. Chen, "Energy harvesting technologies in roadway and bridge for different applications-A comprehensive review," Applied energy, vol. 212, pp.1083-1094, 2018.

[7] Z. Yang, L. Bo, and Z. Li, "Recent progress in human body energy harvesting for smart bioelectronic system," Fundamental Research, vol.1, no. 3, pp. 364-385, 2021.

[8] C. Xu, Y. Song, M. Han, and H. Zhang, "Portable and wearable selfpowered systems based on emerging energy harvesting technology," Microsystems \& Nanoengineering, vol. 7, no. 1, pp. 1-14, 2021.

[9] J. Cui, H. Yoon, and B.D. Youn, "An omnidirectional biomechanical energy harvesting (OBEH) sidewalk block for a self-generative power grid in a smart city," International Journal of Precision Engineering and Manufacturing-Green Technology, vol. 5, no. 4, pp. 507-517, 2018.

[10] H.S. Kim, J-H. Kim, and J. Kim, "A review of piezoelectric energy harvesting based on vibration," International journal of precision engineering and manufacturing, vol. 12, no. 6, pp. 1129-1141, 2011.

[11] M-G. Kang, W-S. Jung, C-Y. Kang, and S-J.Yoon, "Recent progress on PZT based piezoelectric energy harvesting technologies," In Actuators, vol. 5, no. 1, p. 5. Multidisciplinary Digital Publishing Institute, 2016.

[12] Narita, Fumio, and Marina Fox. "A review on piezoelectric, magnetostrictive, and magnetoelectric materials and device technologies for energy harvesting applications." Advanced Engineering Materials 20, no. 5 (2018): 1700743.

[13] Elahi, Hassan, Marco Eugeni, and Paolo Gaudenzi. "A review on mechanisms for piezoelectric-based energy harvesters." Energies 11, no. 7 (2018): 1850.

[14] Priya, Shashank, Hyun-Cheol Song, Yuan Zhou, Ronnie Varghese, Anuj Chopra, Sang-Gook Kim, Isaku Kanno et al. "A review on piezoelectric energy harvesting: materials, methods, and circuits." Energy Harvesting and Systems 4, no. 1 (2019): 3-39.

[15] Wang, Zhong Lin. "Nanogenerators, self-powered systems, blue energy, piezotronics, and piezo-phototronics-A recall on the original thoughts for coining these fields." Nano Energy 54 (2018): 477-483.

[16] Granstrom, Jonathan, Joel Feenstra, Henry A. Sodano, and Kevin Farinholt. "Energy harvesting from a backpack instrumented with piezoelectric shoulder straps." Smart Materials and Structures 16, no. 5 (2007): 1810.

[17] Caliò, Renato, Udaya Bhaskar Rongala, Domenico Camboni, Mario Milazzo, Cesare Stefanini, Gianluca De Petris, and Calogero Maria Oddo. "Piezoelectric energy harvesting solutions." Sensors 14, no. 3 (2014): 4755-4790.

[18] Pillatsch, Pit, Eric M. Yeatman, Andrew S. Holmes, and Paul K. Wright. "Wireless power transfer system for a human motion energy harvester." Sensors and Actuators A: Physical 244 (2016): 77-85.

[19] Fan, Kangqi, Bo Yu, Yingmin Zhu, Zhaohui Liu, and Liansong Wang. "Scavenging energy from the motion of human lower limbs via a piezoelectric energy harvester." International Journal of Modern Physics B 31, no. 7 (2017): 1741011.
[20] Wang, Wei, Junyi Cao, Chris R. Bowen, Shengxi Zhou, and Jing Lin. "Optimum resistance analysis and experimental verification of nonlinear piezoelectric energy harvesting from human motions." Energy 118 (2017): 221-230.

[21] Wang, Xiaoxiong, Wei-Zhi Song, Ming-Hao You, Jun Zhang, Miao Yu, Zhiyong Fan, Seeram Ramakrishna, and Yun-Ze Long. "Bionic singleelectrode electronic skin unit based on piezoelectric nanogenerator." Acs Nano 12, no. 8 (2018): 8588-8596.

[22] Kim, Min-Ook, Soonjae Pyo, Yongkeun Oh, Yunsung Kang, Kyung-Ho Cho, Jungwook Choi, and Jongbaeg Kim. "Flexible and multidirectional piezoelectric energy harvester for the self-powered human motion sensor." Smart Materials and Structures 27, no. 3 (2018): 035001.

[23] Kakihara, R., K. Kariya, Y. Matsushita, T. Yoshimura, and N. Fujimura. "Investigation of piezoelectric energy harvesting from human walking." In Journal of Physics: Conference Series, vol. 1052, no. 1, p. 012113. IOP Publishing, 2018.

[24] Guido, Francesco, Antonio Qualtieri, Luciana Algieri, Enrico Domenico Lemma, Massimo De Vittorio, and Maria Teresa Todaro. "AlN-based flexible piezoelectric skin for energy harvesting from human motion." Microelectronic Engineering 159 (2016): 174-178.

[25] Wu, Yipeng, Jinhao Qiu, Shengpeng Zhou, Hongli Ji, Yang Chen, and Sen Li. "A piezoelectric spring pendulum oscillator used for multidirectional and ultra-low frequency vibration energy harvesting." Applied energy 231 (2018): 600-614.

[26] Qian, Feng, Tian-Bing Xu, and Lei Zuo. "Design, optimization, modeling, and testing of a piezoelectric footwear energy harvester." Energy conversion and management 171 (2018): 1352-1364.

[27] Wang, Sihong, Long Lin, and Zhong Lin Wang. "Nanoscale triboelectric-effect-enabled energy conversion for sustainably powering portable electronics." Nano Letters 12, no. 12 (2012): 6339-6346.

[28] Wang, Zhong Lin. "Triboelectric nanogenerators as new energy technology and self-powered sensors-Principles, problems, and perspectives." Faraday discussions 176 (2015): 447-458.

[29] Wang, Sihong, Long Lin, Yannan Xie, Qingshen Jing, Simiao Niu, and Zhong Lin Wang. "Sliding-triboelectric nanogenerators based on inplane charge-separation mechanism." Nano Letters 13, no. 5 (2013): 2226-2233.

[30] Fan, Feng Ru, Wei Tang, and Zhong Lin Wang. "Flexible nanogenerators for energy harvesting and self-powered electronics." Advanced Materials 28, no. 22 (2016): 4283-4305.

[31] Song, Peiyi, Guang Yang, Tingting Lang, and Ken-Tye Yong. "Nanogenerators for wearable bioelectronics and biodevices." Journal of Physics D: Applied Physics 52, no. 2 (2018): 023002.

[32] Wang, Sihong, Yannan Xie, Simiao Niu, Long Lin, and Zhong Lin Wang. "Freestanding triboelectric-layer-based nanogenerators for harvesting energy from a moving object or human motion in contact and non-contact modes." Advanced materials 26, no. 18 (2014): 2818-2824.

[33] Huang, Tao, Cheng Wang, Hao Yu, Hongzhi Wang, Qinghong Zhang, and Meifang Zhu. "Human walking-driven wearable all-fiber triboelectric nanogenerator containing electrospun polyvinylidene fluoride piezoelectric nanofibers." Nano Energy 14 (2015): 226-235.

[34] Tang, Wei, Tao Jiang, Feng Ru Fan, Ai Fang Yu, Chi Zhang, Xia Cao, and Zhong Lin Wang. "Liquid-metal electrode for high-performance triboelectric nanogenerator at an instantaneous energy conversion efficiency of 70.6\%." Advanced Functional Materials 25, no. 24 (2015): 3718-3725.

[35] Ha, Minjeong, Jonghwa Park, Youngoh Lee, and Hyunhyub Ko. "Triboelectric generators and sensors for self-powered wearable electronics." Acs Nano 9, no. 4 (2015): 3421-3427.

[36] Lin, Zhiming, Jun Chen, Xiaoshi Li, Zhihao Zhou, Keyu Meng, Wei Wei, Jin Yang, and Zhong Lin Wang. "Triboelectric nanogenerator enabled body sensor network for self-powered human heart-rate monitoring." ACS Nano 11, no. 9 (2017): 8830-8837.

[37] Shen, Jiali, Zhaoling Li, Jianyong Yu, and Bin Ding. "Humidityresisting triboelectric nanogenerator for high-performance biomechanical energy harvesting." Nano Energy 40 (2017): 282-288.

[38] Yu, Aifang, Xiong Pu, Rongmei Wen, Mengmeng Liu, Tao Zhou, Ke Zhang, Yang Zhang, Junyi Zhai, Weiguo Hu, and Zhong Lin Wang. 
"Core-shell-yarn-based triboelectric nanogenerator textiles as power cloths." ACS Nano 11, no. 12 (2017): 12764-12771.

[39] Chen, Shu Wen, Xia Cao, Ning Wang, Long Ma, Hui Rui Zhu, Magnus Willander, Yang Jie, and Zhong Lin Wang. "An ultrathin flexible singleelectrode triboelectric-nanogenerator for mechanical energy harvesting and instantaneous force sensing." Advanced Energy Materials 7, no. 1 (2017): 1601255.

[40] Zhang, Zengxing, Kang Du, Xi Chen, Chenyang Xue, and Kaiying Wang. "An air-cushion triboelectric nanogenerator integrated with stretchable electrode for human-motion energy harvesting and monitoring." Nano Energy 53 (2018): 108-115.

[41] Ding, Xiaoheng, Hailin Cao, Xinghong Zhang, Mingyu Li, and Yuntian Liu. "Large scale triboelectric nanogenerator and the self-powered flexible sensor for human sleep monitoring." Sensors 18, no. 6 (2018): 1713.

[42] Ning, Chuan, Lan Tian, Xinya Zhao, Shengxin Xiang, Yingjie Tang, Erjun Liang, and Yanchao Mao. "Washable textile-structured singleelectrode triboelectric nanogenerator for self-powered wearable electronics." Journal of Materials Chemistry A 6, no. 39 (2018): 1914319150.

[43] Song, Yu, Haobin Wang, Xiaoliang Cheng, Guoke Li, Xuexian Chen, Haotian Chen, Liming Miao, Xiaosheng Zhang, and Haixia Zhang. "High-efficiency self-charging smart bracelet for portable electronics." Nano Energy 55 (2019): 29-36.

[44] Xia, Kequan, Zhiyuan Zhu, Hongze Zhang, Chaolin Du, Jiangming Fu, and Zhiwei Xu. "Milk-based triboelectric nanogenerator on paper for harvesting energy from human body motion." Nano Energy 56 (2019): 400-410.

[45] Wang, Lingyun, and Walid A. Daoud. "Highly flexible and transparent polyionic-skin triboelectric nanogenerator for biomechanical motion harvesting." Advanced Energy Materials 9, no. 5 (2019): 1803183.

[46] Xiong, Jiaqing, Peng Cui, Xiaoliang Chen, Jiangxin Wang, Kaushik Parida, Meng-Fang Lin, and Pooi See Lee. "Skin-touch-actuated textilebased triboelectric nanogenerator with black phosphorus for durable biomechanical energy harvesting." Nature Communications 9, no. 1 (2018): 1-9.

[47] Saha, C. R., T. O'donnell, N. Wang, and P. McCloskey. "Electromagnetic generator for harvesting energy from human motion." Sensors and Actuators A: Physical 147, no. 1 (2008): 248-253.

[48] Dai, Dan, and Jing Liu. "Hip-mounted electromagnetic generator to harvest energy from human motion." Frontiers in Energy 8, no. 2 (2014): 173-181.

[49] Luciano, V., E. Sardini, M. Serpelloni, and G. Baronio. "An energy harvesting converter to power sensorized total human knee prosthesis." Measurement Science and Technology 25, no. 2 (2014): 025702.

[50] Zhang, Qian, Yufeng Wang, and Eun Sok Kim. "Power generation from human body motion through magnet and coil arrays with magnetic spring." Journal of Applied Physics 115, no. 6 (2014): 064908.

[51] Dinulovic, Dragan, Michael Brooks, Martin Haug, and Tomislav Petrovic. "Rotational electromagnetic energy harvesting system." Physics Procedia 75 (2015): 1244-1251.

[52] Quan, Ting, Xue Wang, Zhong Lin Wang, and Ya Yang. "Hybridized electromagnetic-triboelectric nanogenerator for a self-powered electronic watch." Acs Nano 9, no. 12 (2015): 12301-12310.

[53] Bendame, Mohamed, Eihab Abdel-Rahman, and Mostafa Soliman. "Electromagnetic impact vibration energy harvesters." In Structural nonlinear dynamics and diagnosis, pp. 29-58. Springer, Cham, 2015.

[54] Niroomand, Mehdi, and Hamid Reza Foroughi. "A rotary electromagnetic microgenerator for energy harvesting from human motions." Journal of applied research and technology 14, no. 4 (2016): 259-267.

[55] Geisler, M., S. Boisseau, M. Perez, I. Ait-Ali, and S. Perraud. "Scaling effects in a nonlinear electromagnetic energy harvester for wearable sensors." In Journal of Physics: Conference Series, vol. 773, no. 1, p. 012044. IOP Publishing, 2016.

[56] Brunner, Stephan, Maximilian Gerst, and Christian Pylatiuk. "Design of a body energy harvesting system for the upper extremity." Current Directions in Biomedical Engineering 3, no. 2 (2017): 331-334.

[57] Halim, M. A., R. Rantz, Q. Zhang, L. Gu, K. Yang, and S. Roundy. "Electromagnetic energy harvesting from swing-arm motion using rotational eccentric mass structure." In 2017 19th international conference on solid-state sensors, actuators, and microsystems (TRANSDUCERS), pp. 1863-1866. IEEE, 2017.

[58] Halim, M. A., R. Rantz, Q. Zhang, L. Gu, K. Yang, and S. J. A. E. Roundy. "An electromagnetic rotational energy harvester using sprung eccentric rotor, driven by pseudo-walking motion." Applied Energy 217 (2018): 66-74.

[59] Zhang, Ying, Junyi Cao, Hongyu Zhu, and Yaguo Lei. "Design, modeling and experimental verification of circular Halbach electromagnetic energy harvesting from bearing motion." Energy conversion and management 180 (2019): 811-821.

[60] Fan, Kangqi, Yiwei Zhang, Haiyan Liu, Meiling Cai, and Qinxue Tan. "A nonlinear two-degree-of-freedom electromagnetic energy harvester for ultra-low frequency vibrations and human body motions." Renewable Energy 138 (2019): 292-302.

[61] Leonov, Vladimir. "Energy harvesting for self-powered wearable devices." In Wearable monitoring systems, pp. 27-49. Springer, Boston, MA, 2011.

[62] Xie, Longhan, and Mingjing Cai. "Human motion: Sustainable power for wearable electronics." IEEE Pervasive Computing 13, no. 4 (2014): 42-49.

[63] Ghomian, Taher, and Shahab Mehraeen. "Survey of energy scavenging for wearable and implantable devices." Energy 178 (2019): 33-49.

[64] Khalifa, Sara, Guohao Lan, Mahbub Hassan, Aruna Seneviratne, and Sajal K. Das. "Harke: Human activity recognition from kinetic energy harvesting data in wearable devices." IEEE Transactions on Mobile Computing 17, no. 6 (2017): 1353-1368.

[65] Dagdeviren, Canan, Zhou Li, and Zhong Lin Wang. "Energy harvesting from the animal/human body for self-powered electronics." Annual review of biomedical engineering 19 (2017): 85-108.

[66] Pavelková, Radka, David Vala, and Kateřina Gecová. "Energy harvesting systems using human body motion." IFAC-PapersOnLine 51, no. 6 (2018): 36-41.

[67] Invernizzi, F., S. Dulio, M. Patrini, G. Guizzetti, and P. Mustarelli. "Energy harvesting from human motion: materials and techniques." Chemical Society Reviews 45, no. 20 (2016): 5455-5473.

[68] Halim, Miah A., Hyunok Cho, Md Salauddin, and Jae Y. Park. "A miniaturized electromagnetic vibration energy harvester using fluxguided magnet stacks for human-body-induced motion." Sensors and Actuators A: Physical 249 (2016): 23-31.

[69] Wu, Shuai, Patrick Chi-Kwong Luk, Chunfang Li, Xiangyu Zhao, Zongxia Jiao, and Yaoxing Shang. "An electromagnetic wearable 3-DoF resonance human body motion energy harvester using ferrofluid as a lubricant." Applied Energy 197 (2017): 364-374.

[70] Fan, Kangqi, Meiling Cai, Haiyan Liu, and Yiwei Zhang. "Capturing energy from ultra-low frequency vibrations and human motion through a monostable electromagnetic energy harvester." Energy 169 (2019): 356368.

[71] Tian, Jingjing, Rui Shi, Zhuo Liu, Han Ouyang, Min Yu, Chaochao Zhao, Yang Zou, Dongjie Jiang, Jingshuang Zhang, and Zhou Li. "Selfpowered implantable electrical stimulator for osteoblasts' proliferation and differentiation." Nano Energy 59 (2019): 705-714.

[72] Yang, Ye, Hong Pan, Guangzhong Xie, Yadong Jiang, Chunxu Chen, Yuanjie Su, Yang Wang, and Huiling Tai. "Flexible piezoelectric pressure sensor based on polydopamine-modified BaTiO3/PVDF composite film for human motion monitoring." Sensors and Actuators A: Physical 301 (2020): 111789. 